\title{
Reduced miR-200b and miR-200c expression contributes to abnormal hepatic lipid accumulation by stimulating JUN expression and activating the transcription of srebp1
}

\author{
Jun Guo ${ }^{1,3, *}$, Weiwei Fang ${ }^{1,2, *}$, Libo Sun ${ }^{4}$, Yonggang Lu ${ }^{1}$, Lin Dou ${ }^{1}$, Xiuqing Huang ${ }^{1}$, \\ Mingxiao Sun ${ }^{1}$, Cheng Pang ${ }^{1,2}$, Jing $\mathbf{Q u}^{5,6}$, Guanghui $\mathrm{Liu}^{3,6}$, Jian $\mathrm{Li}^{1,2}$ \\ ${ }^{1}$ The Key Laboratory of Geriatrics, Beijing Hospital and Beijing Institute of Geriatrics, Ministry of Health, Beijing 100730, China \\ ${ }^{2}$ Graduate School of Peking Union Medical College and Chinese Academy of Medical Sciences, Beijing 100730, China \\ ${ }^{3}$ National Laboratory of Biomacromolecules, Institute of Biophysics, University of Chinese Academy of Sciences, Chinese \\ Academy of Sciences, Beijing 100101, China \\ ${ }^{4}$ Department of Hepatobiliay Surgery and You-An Liver Transplantation Center, Beijing You-An Hospital, Capital Medical \\ University, Beijing 100069, China \\ ${ }^{5}$ State Key Laboratory of Stem Cell and Reproductive Biology, Institute of Zoology, Chinese Academy of Sciences, Beijing \\ 100101, China \\ ${ }^{6}$ University of Chinese Academy of Sciences, Beijing 100049, China \\ *These authors contributed equally to this work \\ Correspondence to: Jian Li, email: lijian@bjhmoh.cn \\ Guanghui Liu, email: ghliu@ibp.ac.cn \\ Jing Qu, email: qujing@ioz.ac.cn
}

Keywords: miR-200b, miR-200c, lipogenesis, JUN, SREBP1

Received: September 22, 2015

Accepted: April 22, 2016

Published: May 05, 2016

\section{ABSTRACT}

Previous studies indicated that miR-200s participated in IL-6-induced hepatic insulin resistance. However, the role of $\mathbf{m i R - 2 0 0 s}$ in hepatic lipid accumulation has not been elucidated. Here we found that miR-200b and miR-200c were reduced in the steatotic livers of mice fed a high-fat diet (HFD) and patients with nonalcoholic fatty liver disease. This down-regulation was accompanied by an increase in the expression of lipogenic proteins such as sterol regulatory element-binding protein 1 (SREBP1) and fatty acid synthase (FAS). The suppression of miR-200b and miR-200c in Hep1-6 and NCTC1469 hepatocytes enhanced intracellular triglyceride levels, which were associated with increased SREBP-1 and FAS protein levels. In contrast, the overexpression of miR-200b and miR-200c suppressed lipid accumulation and reduced the expression of SREBP1 and FAS in Hep1-6 and NCTC1469 cells transfected with miR-200b or miR-200c mimics. Importantly, the up-regulation of miR-200b and miR-200c could reverse oleic acid/palmitic acid-induced lipid accumulation in hepatocytes. A luciferase reporter assay identified that miR-200b and miR-200c could directly bind the 3'UTR of jun. JUN activated the transcription of srebp1 to increase lipid accumulation. The data also demonstrated that increased miR-200b and miR-200c expression might be associated with sitagliptin-reduced hepatic lipid accumulation in mice fed a HFD. These findings suggest, for the first time, that reduced miR-200b and miR-200c expression contributes to abnormal hepatic lipid accumulation by stimulating JUN expression and activating the transcription of srebp1.

\section{INTRODUCTION}

Obesity is a global health problem that causes a series of pathological disorders including nonalcoholic fatty liver disease (NAFLD), type 2 diabetes and cardiovascular disease [1-3]. Abnormal lipid accumulation in the liver may result in hepatic steatosis and nonalcoholic steatohepatitis (NASH) $[4,5]$. Great advances have been made in defining the pathogenesis of NAFLD. However, the specific molecular mechanism responsible for the disease is still poorly understood.

Liver lipid homeostasis is correlated with different processes including the uptake, synthesis, storage and secretion of lipids [6]. As major transcription factors, 
sterol regulatory element-binding proteins (SREBPs) have been reported to widely regulate lipogenic gene expression [7]. The SREBP family includes SREBP-1a, SREBP-1c and SREBP-2, all of which are transcribed from two distinct genes, srebpl and srebp2. Two alternatively spliced transcripts, $s r e b p-1 a$ and $s r e b p$ $1 c$, are expressed from various promoters [8]. SREBP1c mainly stimulates proteins involved in fatty acid metabolism such as fatty acid synthase (FAS) $[9,10]$. In comparison, SREBP-2 and SREBP-1a mainly participate in cholesterol metabolism [11].

MicroRNAs (miRs) are small, non-coding RNAs that are important post-transcriptional regulators of gene expression. By binding to the $3^{\prime}$ untranslated region (3'UTR) of their target genes, they globally repress gene expression [14]. miRs have been shown to be involved in a wide variety of physiological and psychological processes $[15,16]$. miR-34a was increased in patients with NAFLD and in mice fed a high-fat diet (FHDfed mice) [17]. miR-33a and miR-33b were found to globally regulate lipid metabolism by targeting numerous lipid metabolism-associated genes including abcal, crot, cptla, hadhb and ampk $\alpha$ [19]. The miR-200 family, including miR-200a, miR-200b, miR-200c, miR141 and miR-429, has been reported to be dysregulated in several types of cancers including gastric cancer, breast cancer and bladder cancer [20-22]. In a previous study, we demonstrated that the reduction of miR-200a, miR-200b and miR-200c in hepatocytes contributed to IL-induced insulin resistance [23]. However, the role of the miR-200 family in hepatic lipid metabolism remains unknown. The aim of this study was to explore the potential role of the miR-200 family in the regulation of hepatic lipid metabolism. The findings suggest that the reduced expression of miR-200b and miR-200c participated in abnormal hepatic lipid accumulation by stimulating JUN expression and activating the transcription of srebp1.

\section{RESULTS}

\section{The levels of miR-200b and miR-200c are reduced in the steatotic livers of HFD-fed mice and NAFLD patients}

Oil red $\mathrm{O}$ and $\mathrm{H} \& \mathrm{E}$ staining showed abnormal lipid accumulation in the livers of mice fed a HFD (Figure 1A). The livers of HFD-fed mice displayed excessive amounts of triglycerides (Figure 1B). Moreover, the expression of lipogenic genes including srebpl and fas was significantly increased in the livers of HFD-fed mice (Figure 1C). To identify the potential role of the miR-200 family in lipid metabolism, relative expression patterns were analyzed in the steatotic livers of HFD-fed mice and NAFLD patients. As shown in Figure 1D, the levels of miR$200 \mathrm{~b}$ and miR-200c, but not the levels of other members of the miR-200 family including miR-200a, miR-141 and miR-429, were obviously reduced in the livers of HFD-fed mice. As shown in Table 1, the age and gender distribution were similar between the healthy controls and NAFLD patients. Characteristics such as BMI, waist circumference and triglyceride levels were significantly higher in the NAFLD patients compared with the healthy controls. H\&E staining showed that the cytoplasm of the NAFLD patient hepatocytes was filled with lipid droplets (Figure 1E). Importantly, the expression of miR-200b and miR-200c was suppressed in the livers of NAFLD patients (Figure 1F), and the levels of lipogenic proteins such as SREBP1 and FAS were elevated compared with the healthy controls (Figure 1G). These data suggest that miR-200b and miR-200c may be involved in hepatic lipogenesis.

\section{Reduced miR-200b and miR-200c expression contributes to abnormal lipid accumulation in Hep1-6 and NCTC1469 cells}

To determine whether miR-200b and miR-200c are involved in abnormal hepatic lipid accumulation, the expression of miR-200b and miR-200c was suppressed in two murine liver cell lines, Hep1-6 and NCTC1469, by transfection with miR-200b and miR-200c inhibitors. As shown in Figure $2 \mathrm{~A}$ and $2 \mathrm{~B}$, the transfection of both Hep1-6 and NCTC1469 cells with miR-200b and miR-200c inhibitors resulted in a significant increase in intracellular triglyceride levels. This in turn was associated with elevated SREBP-1 and FAS protein levels (Figure 2C and 2D). This finding suggests that reduced miR-200b and miR-200c expression might contribute to abnormal lipid accumulation in hepatocytes. In contrast, the over-expression of miR-200b and miR-200c significantly reduced lipid accumulation in Hep1-6 and NCTC1469 cells transfected with miR-200b and miR-200c mimics (Figure 2E and 2F). This was accompanied by decreased SREBP1 and FAS levels (Figure $2 \mathrm{G}$ and $2 \mathrm{H}$ ), indicating that miR-200b and miR-200c mimics have a suppressive role in lipid accumulation.

\section{The over-expression of miR-200b and miR-200c reverses oleic acid/palmitic acid-induced lipid accumulation in hepatocytes}

To further investigate the suppressive role of miR-200b and miR-200c mimics in lipid accumulation, Hep1-6 and NCTC1469 cells were pre-treated with a mixture of oleic acid and palmitic acid (2:1, M/M) for $24 \mathrm{~h}$. Oil red $\mathrm{O}$ staining revealed that pre-treatment with oleic acid/palmitic acid (O/P) significantly promoted lipid accumulation in Hep1-6 and NCTC1469 cells (Figure $3 \mathrm{~A}$ and 3B). Interestingly, the transfection of both Hep1-6 and NCTC1469 cells with miR-200b and 
miR-200c mimics partially reversed the formation of the $\mathrm{O} / \mathrm{P}$-induced lipid droplets (Figure $3 \mathrm{~A}$ and $3 \mathrm{~B}$ ) and the elevation of SREBP1 and FAS levels (Figure 3C and 3D).

\section{jun is a target gene of miR-200b and miR-200c}

To explore the specific mechanism by which miR-200b and miR-200c regulate lipogenesis in hepatocytes, miR-200b and miR-200c target genes were identified through the bioinformatics websites miRBase, TargetScan and RNA22. Unfortunately, no target genes directly involved in lipid metabolism were identified. Instead, a transcription factor, jun, was predicted to be a target gene of miR-200b and miR-200c (Figure 4A). To verify this finding, the 3'UTR of jun was cloned into the pmirGLO plasmid. As shown in Figure 4B, the miR-200b and miR-200c mimics significantly reduced the relative luciferase activity of pmirGLO-jun-3'UTR, indicating a direct binding. Furthermore, the mutant of jun 3'UTR was inserted into the pmirGLO plasmid, but overexpression of miR-200b and miR-200c could not decrease the relative luciferase activity of pmirGLO-jun-3'UTR-Mut (Figure 4B). Western blotting showed that the over-expression of miR-200b and miR-200c obviously reduced the level of JUN protein (Figure 4C), while the inhibition of miR-200b and miR200c significantly elevated the expression of JUN in Hep16 cells (Figure 4D), demonstrating that jun is a target gene of miR-200b and miR-200c.

\section{JUN stimulates lipid accumulation by activating the transcription of srebp1}

As a transcription factor, JUN participates in cell proliferation, cell survival, apoptosis and tumorigenesis by activating gene expression $[24,25]$. In the present study, two putative JUN binding sites were identified in the promoter region of the mouse srebpl gene. To confirm the potential binding sites, the regions of the srebpl promoter containing both of the binding sites, one of the binding sites (pGL3-srebp1-S1) or neither of the binding sites (pGL3-srebp1-S2) were cloned into the pGL3 vector (Figure 5A). HEK293 cells were then transfected with siRNA specifically targeting jun (si-jun-1 or si- jun-2) along with the luciferase reporter plasmids containing srebp1, srebp1-S1 or srebp1-S2. The results indicated that the knockdown of jun decreased the relative luciferase activity of pGL3-srebp1 and pGL3-srebp1-S1, but had no effect on pGL3-srebp1-S2 (Figure 5B). Furthermore,

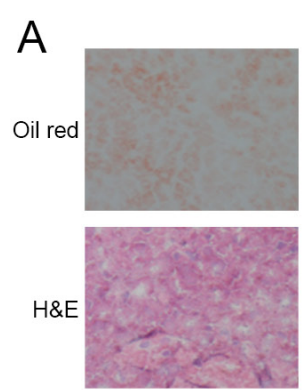

C57

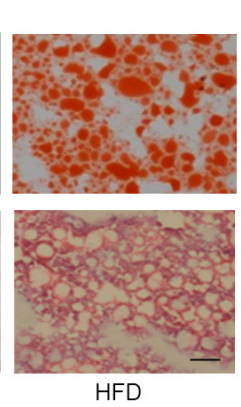

B

C
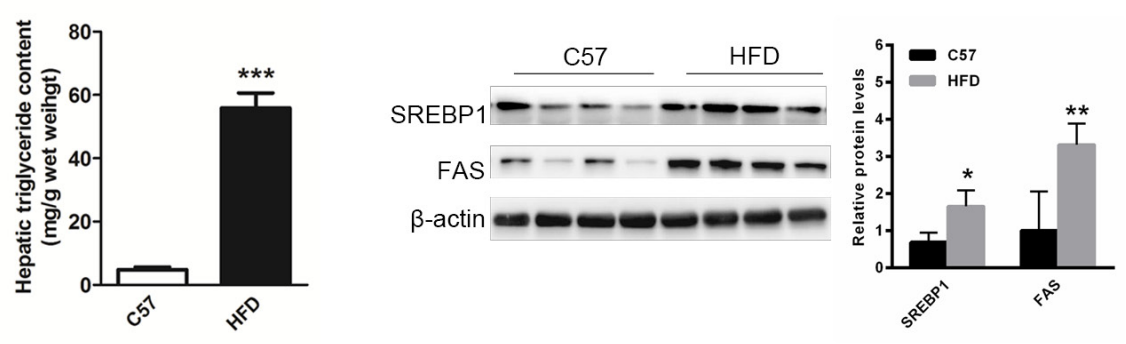

D

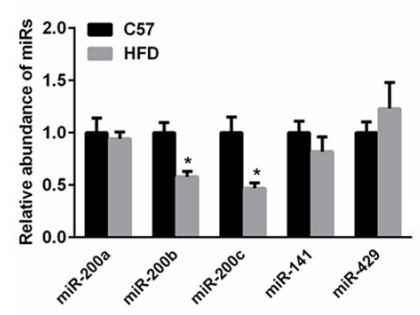

E

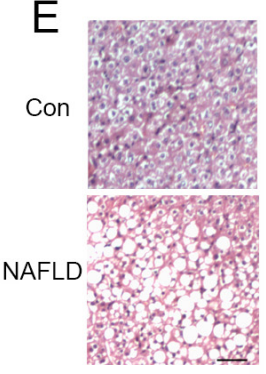

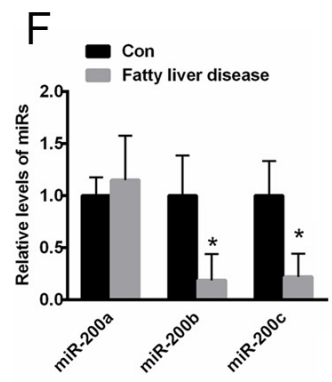

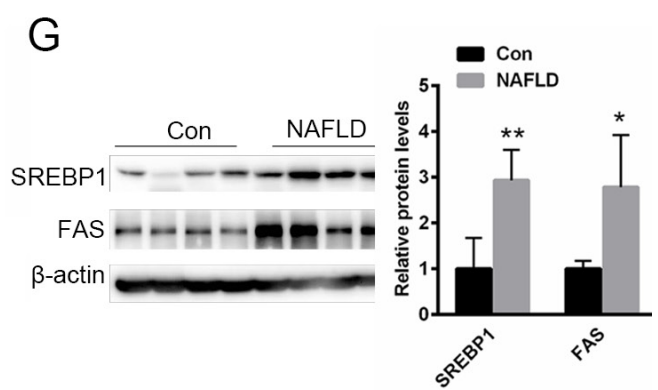

Figure 1: The levels of miR-200b and miR-200c are reduced in the steatotic livers of NAFLD patients and mice fed a HFD. (A) Oil red O and H\&E staining of the livers of HFD-fed mice. (B) The measurement of triglyceride levels in the livers of HFD-fed mice. (C) Western blots showing the expression of the lipogenic proteins SREBP1 and FAS. (D, F) Real-time reverse-transcription PCR showing the relative expression patterns of miR-200 family members including miR-200b, miR-200c, miR-200a, miR-141 and miR-429 in the steatotic livers of HFD-fed mice $(n=5)$ or in the livers of NAFLD patients and healthy subjects $(n=11)$. (E) H\&E staining of the livers of NAFLD patients. (G) Western blots showing the expression of SREBP1 and FAS in the livers of NAFLD patients. The data represent the mean \pm SEM. $* P<0.05$ and $* * P<0.01$ versus the control. The bar represents $25 \mu \mathrm{m}$. 
Table 1: Clinical and biochemical characteristics of healthy controls and patients with nonalcoholic fatty liver disease1 $n(\%)$

\begin{tabular}{|c|c|c|c|}
\hline Characteristic & Control $(n=10)$ & NAFLD $(n=10)$ & $P$ value \\
\hline Gender (males/females) & $6 / 5$ & $6 / 5$ & - \\
\hline Age (yr) & $43.0 \pm 8.3$ & $42.5 \pm 9.8$ & 0.906048 \\
\hline $\operatorname{BMI}\left(\mathrm{kg} / \mathrm{m}^{2}\right)$ & $23.0 \pm 4.9$ & $27.0 \pm 2.9$ & 0.029506 \\
\hline Smoking & no & no & - \\
\hline Waist circumference $(\mathrm{cm})$ & $77.4 \pm 9.6$ & $93.1 \pm 8.6$ & 0.001175 \\
\hline Diabetes Mellitus & no & no & - \\
\hline Metabolic syndrome & no & no & - \\
\hline Hypertension & no & no & - \\
\hline Systolic blood pressure $(\mathrm{mmHg})$ & $108.2 \pm 7.5$ & $114.9 \pm 7.5$ & 0.045535 \\
\hline Diastolic blood pressure (mmHg) & $73.3 \pm 5.5$ & $76.8 \pm 5.5$ & 0.138200 \\
\hline AST median (min-max, U/L) & $16.7(13-24)$ & $18.1(12-26)$ & 0.410204 \\
\hline ALT median(min-max, U/L) & $13.8(8-23)$ & $21.7(11-38)$ & 0.024995 \\
\hline Total cholesterol $(\mathrm{mmol} / \mathrm{L})$ & $4.5 \pm 0.4$ & $5.0 \pm 1.1$ & 0.129345 \\
\hline HDL-cholesterol $(\mathrm{mmol} / \mathrm{L})$ & $1.5 \pm 0.3$ & $1.3 \pm 0.2$ & 0.090324 \\
\hline LDL-cholesterol (mmol/L) & $2.0 \pm 0.7$ & $2.9 \pm 1.3$ & 0.074496 \\
\hline Triglycerides $(\mathrm{mmol} / \mathrm{L})$ & $0.8 \pm 0.3$ & $2.3 \pm 1.7$ & 0.007739 \\
\hline
\end{tabular}

a ChIP assay revealed that the knockdown of jun in Hep1-6 cells reduced the binding between JUN and the srebp1 promoter, while the over-expression of JUN enhanced it (Figure 5C). The knockdown of jun reduced SREBP1 and FAS protein levels (Figure 5D and 5E) as well as lipid accumulation (Figure 5F) in both Hep1-6 and NCTC1469 cells. More importantly, the knockdown of jun could partially ameliorate the miR-200b and miR-200c inhibition-induced increased SREBP1 and FAS levels in Hep1-6 cells (Figure 5G). Taken together, these data suggest that miR-200b and miR-200c are involved in lipogenesis through stimulation of JUN expression and the subsequent activation of srebp1 transcription.

\section{The up-regulation of miR-200b and miR-200c expression in the liver prevents hepatic lipid accumulation in HFD-fed mice}

To determine whether miR-200b and miR-200c are involved in hepatic lipid accumulation in vivo, miR-200b and miR-200c were over-expressed in the livers of HFD-fed mice through the use of recombinant adenovirus expressing miR-200b and miR-200c mimics (Ad-miR-200b \& Ad-miR-200c), respectively. Seven days after administration of Ad-miR-200b and Ad-
miR-200c to the mice by tail vein injection, the hepatic levels of miR-200b and miR-200c were elevated by 3.43- and 2.93-fold, respectively (Figure 6A). More importantly, this enhancement of hepatic miR-200b and miR-200c expression led to a significant reduction in liver weight and in the liver weight-to-body weight ratio (Figure 6B, 6C). H\&E and Oil Red $\mathrm{O}$ staining revealed decreased hepatic lipid deposition in these animals (Figure 6D). Of note, the hepatic triglyceride content was significantly reduced (Figure 6E), and this was associated with decreased levels of JUN, SREBP-1 and FAS protein in the liver (Figure 6F). These results suggest that the up-regulation of miR-200b and $\mathrm{miR}-200 \mathrm{c}$ expression in the liver may prevent the accumulation of hepatic triglycerides in HFD-fed mice by suppressing JUN-SREBP1-stimulated lipogenesis.

\section{Elevated miR-200b and miR-200c expression is associated with sitagliptin-reduced hepatic lipid accumulation in HFD-fed mice}

Studies have suggested that sitagliptin reduces hepatic lipid accumulation [26, 27]. However, the specific mechanism has not been fully elucidated. In the present study, Hep1-6 cells were pre-treated with 
a mixture of oleic acid/palmitic acid $(2: 1, \mathrm{M} / \mathrm{M})$ for $24 \mathrm{~h}$ and then treated with $1 \mu \mathrm{M}$ sitagliptin for $24 \mathrm{~h}$. Real-time reverse-transcription PCR indicated that sitagliptin rescued the oleic acid/palmitic acid-induced reduction of $\mathrm{miR}-200 \mathrm{~b}$ and $\mathrm{miR}-200 \mathrm{c}$ expression in Hep1-6 cells (Figure 7A). The study was further extended to HFD-fed mice. Interestingly, miR-200b and miR-200c were enhanced in the livers of HFD-fed mice treated with sitagliptin (Figure 7B). Oil red $\mathrm{O}$ and H\&E staining showed that hepatic accumulation was improved when HFD-fed mice were administered $3 \mathrm{mg} /$ $\mathrm{kg}$ /day sitagliptin via i.g. for 8 weeks compared with the vehicle control (Figure 7C). Hepatic triglyceride contents (Figure 7D) and SREBP1 expression levels (Figure 7E) were also reduced in sitagliptin-treated,
HFD-fed mice. These data indicated that an increase in miR-200b and miR-200c expression might be associated with the sitagliptin-induced reversal of hepatic lipid accumulation in HFD-fed mice.

To further assess the requirement of miR-200 in sitagliptin's effect, miR-200b and miR-200c were knocked down in the Hep1-6 cells pre-treated with a mixture of oleic acid/palmitic acid and treated with sitagliptin. We found that sitagliptin treatment significantly decreased the expression of SREBP1 and FAS. More importantly, we demonstrated that down-regulation of miR-200b and miR-200c could partially reverse sitagliptin-induced decreased levels of SREBP1 and FAS, and reduced contents of intracellular triglyceride in Hep1-6 cells (Figure 7F, 7G).
A

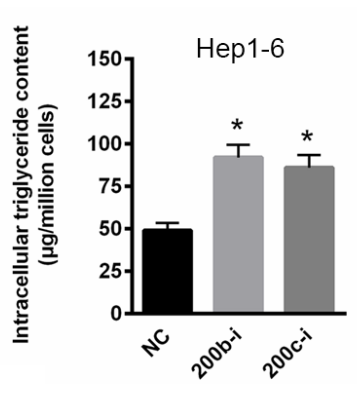

$\mathrm{D}$

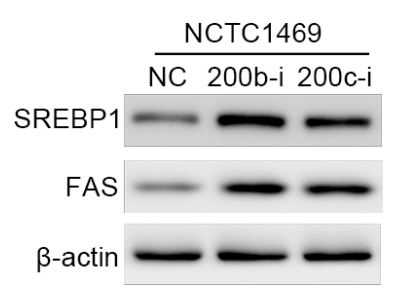

$\mathrm{F}$

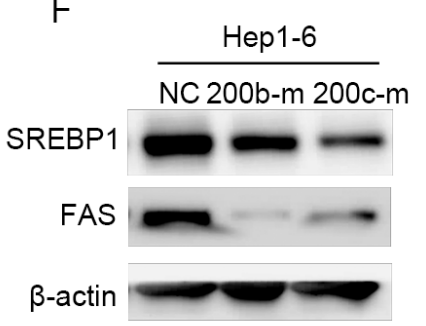

B
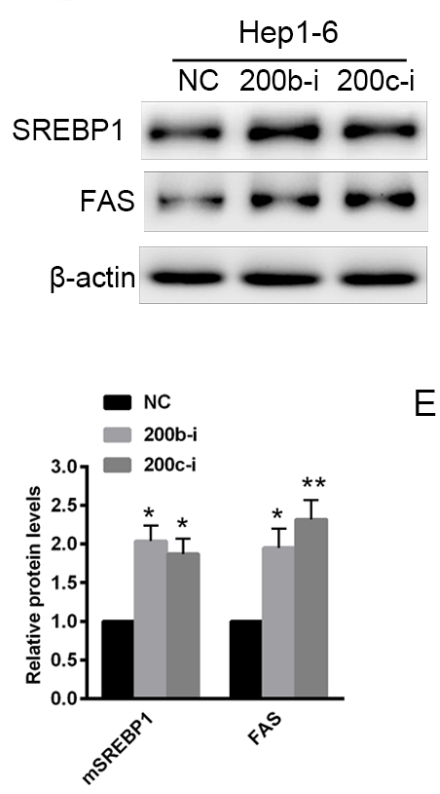

E

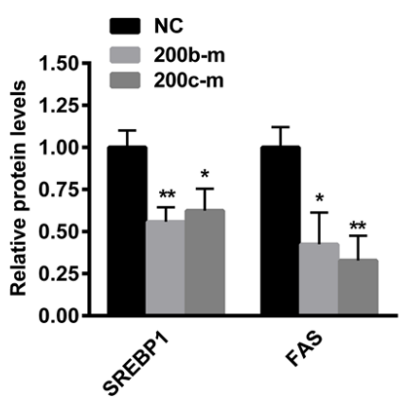

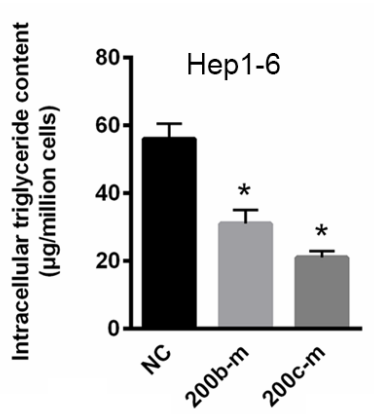

$\mathrm{H}$
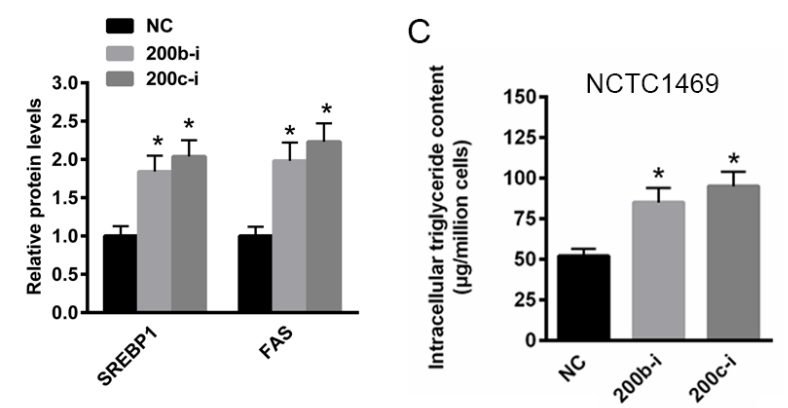

G
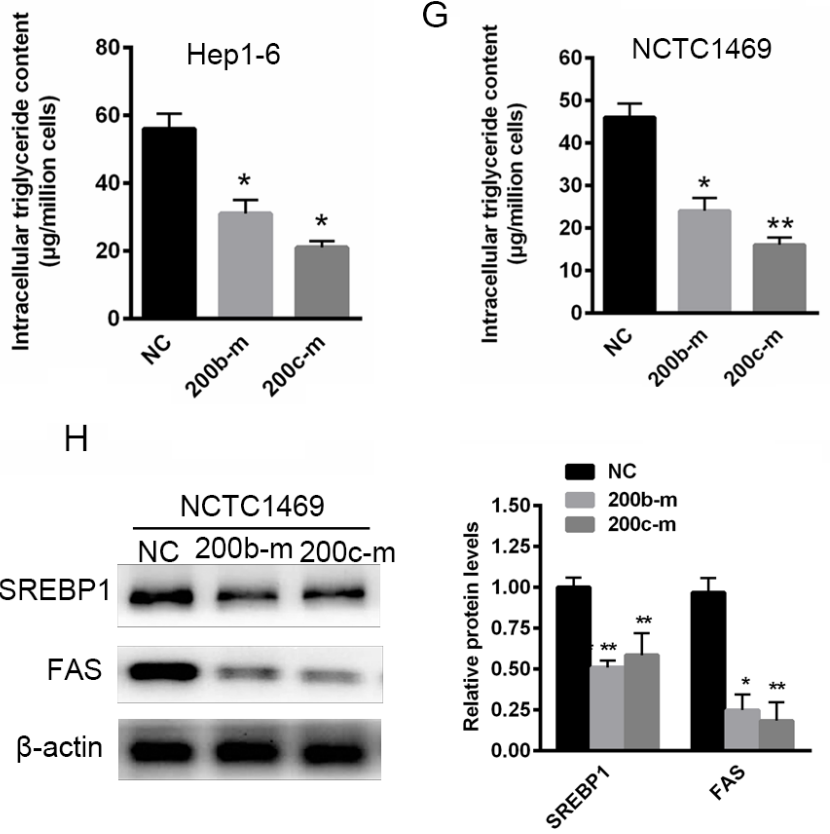

Figure 2: Reduced miR-200b and miR-200c expression contributes to abnormal lipid accumulation in Hep1-6 and NCTC1469 cells. (A, B) The measurement of triglyceride levels in Hep1-6 and NCTC1469 murine liver cells transfected with miR-200b and miR-200c inhibitors. (C, D) Western blots showing the expression of SREBP1 and FAS in Hep1-6 and NCTC1469 cells transfected with miR-200b and miR-200c inhibitors. (E, F) The measurement of triglyceride levels in Hep1-6 and NCTC1469 cells transfected with miR-200b and miR-200c mimics. (G, H) Western blots showing the expression of SREBP1 and FAS in Hep1-6 and NCTC1469 cells transfected with miR-200b and miR-200c mimics. The data represent the mean $\pm \mathrm{SEM}$ of three independent experiments. ${ }^{*} P<0.05$ and $* * P<0.01$ versus the control. 

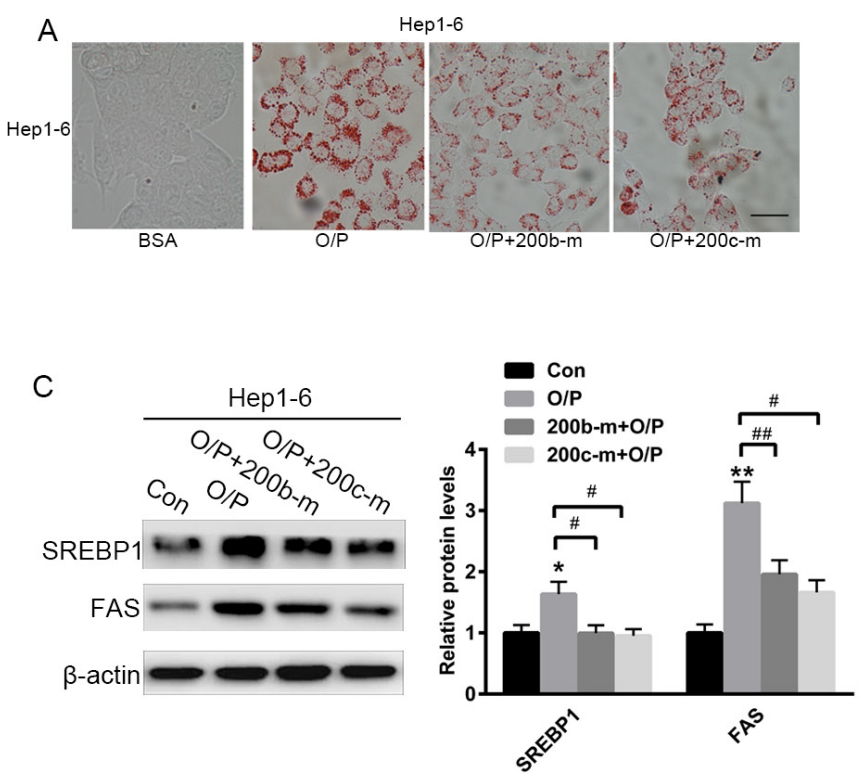
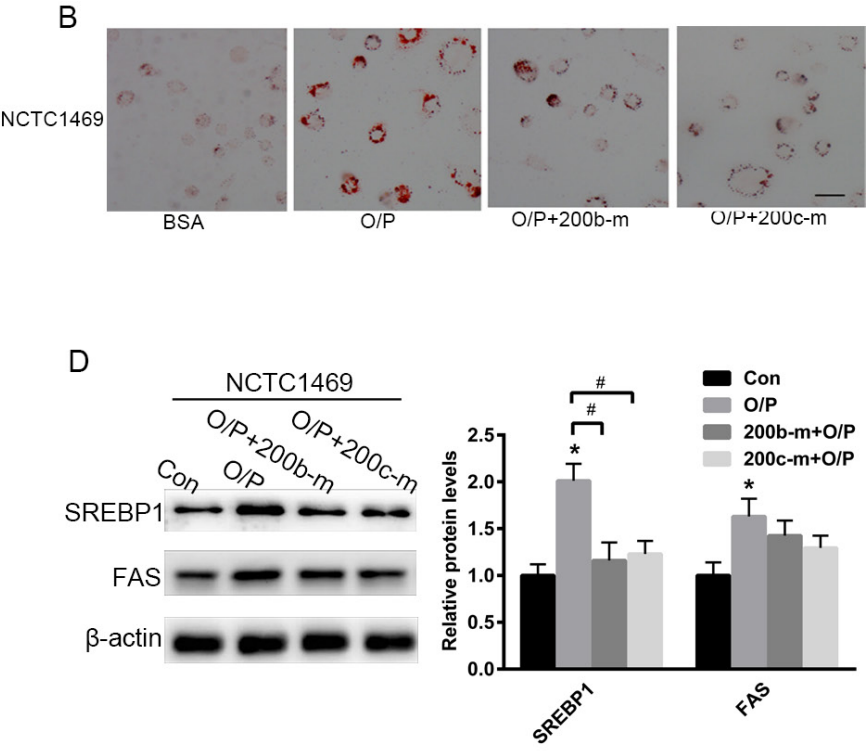

Figure 3: The over-expression of miR-200b and miR-200c reverses oleic acid/palmitic acid-induced lipid accumulation in hepatocytes. (A, B) Oil red O staining of Hep1-6 and NCTC1469 cells pre-treated with a mixture of oleic acid/palmitic acid $(2: 1, \mathrm{M} / \mathrm{M})$ for $24 \mathrm{~h}$. (C, D) Western blots showing the expression of SREBP1 and FAS in Hep1-6 and NCTC1469 cells pre-treated with a mixture of oleic acid/palmitic acid $(2: 1, \mathrm{M} / \mathrm{M})$ for $24 \mathrm{~h}$ and then transfected with miR-200b and miR-200c mimics. The data represent the mean \pm SEM of three independent experiments. ${ }^{*} P<0.05$ and ${ }^{*} P<0.01$ versus the control; ${ }^{*} P<0.05$ and ${ }^{\# \#} P<0.01$ versus O/P. The bar represents $10 \mu \mathrm{m}$.

A

Mouse jun 3'UTR

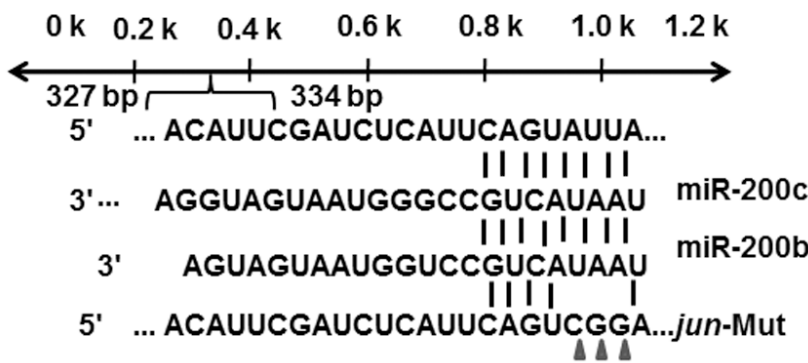

C

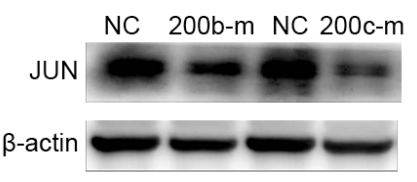

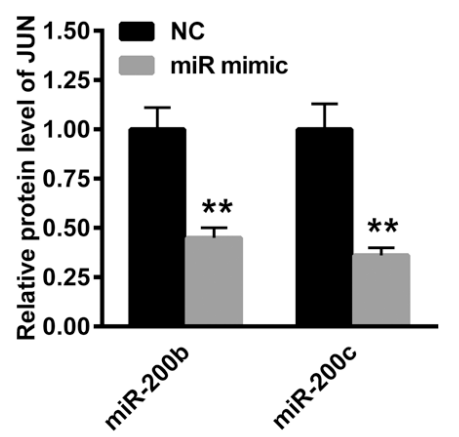

B
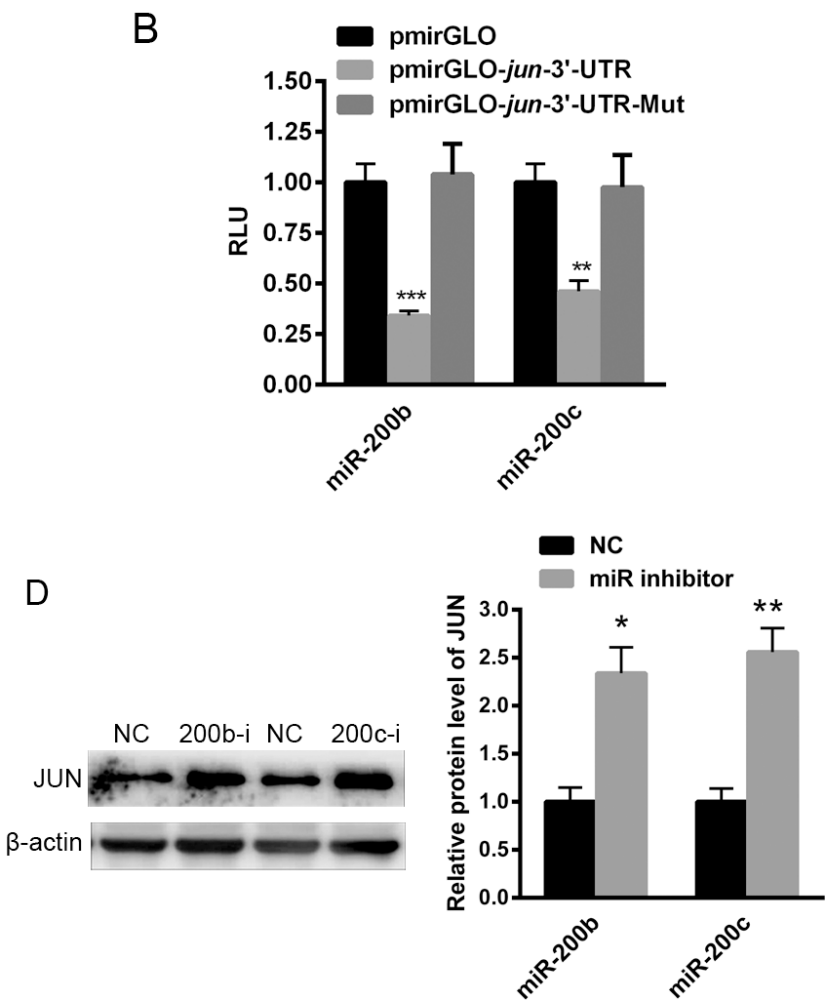

Figure 4: miR-200b and miR-200c bind to the 3'UTR of jun. (A) TargetScan-predicted miR-200b and miR-200c bind to the $3^{\prime} \mathrm{UTR}$ of jun. (B) The luciferase reporter assay used to determine the effects of miR-200b and miR-200c on the relative luciferase activity of pmirGLO-jun-3'UTR. (C) Western blots showing the expression of the JUN protein in Hep1-6 cells transfected with miR-200b and miR-200c mimics. (D) Western blots showing the expression of the JUN protein in Hep1-6 cells transfected with miR-200b and miR-200c inhibitors. The data represent the mean \pm SEM of three independent experiments. ${ }^{*} P<0.05$ and ${ }^{*} P<0.01$ versus the control. 


\section{DISCUSSION}

NAFLD is widely recognized as the most common cause of liver damage. It is characterized by abnormal fat accumulation in the liver and may eventually progress to nonalcoholic steatohepatitis (NASH) with progressive fibrosis [28]. Although great advances have been made in elucidating the pathogenesis of NAFLD, the underlying mechanism is still largely unknown. Emerging evidence suggests that miRNAs are involved in the signaling pathways of NAFLD. Previously, we examined miRNA expression in the livers of $\mathrm{db} / \mathrm{db}$ mice and found that the expression of the miR-200 family members miR-200a, miR-200b and miR-200c was reduced in response to hepatic insulin resistance [23]. The miR-200 family has been extensively studied in different tumors [29-31]. Researchers demonstrated that the miR-200 family influences cancer development and progression by targeting various important signaling factors. However, few studies have been conducted on metabolic disorders. Our previous study demonstrated that the down-regulation of miR-200s following treatment with IL-6 could reduce the activation of the PI3K/AKT/GSK signaling pathway by targeting FOG2 [23]. In this study, we found that only miR-200b and miR-200c were decreased in the livers of NAFLD patients and mice fed a HFD. Additional experiments conducted in Hep1-6 and NCTC1469 cells revealed that the reduced expression of miR-200b and miR-200c led to abnormal lipid accumulation in hepatocytes, whereas the up-regulation of miR-200b and
A

srebp 1 promoter DNA fragments

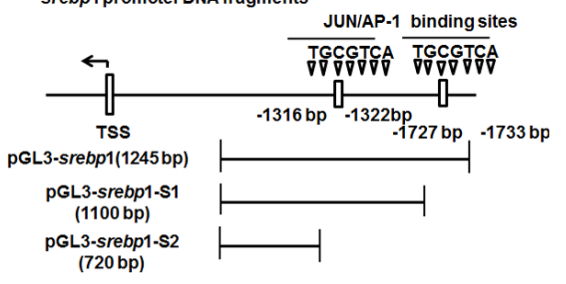

B
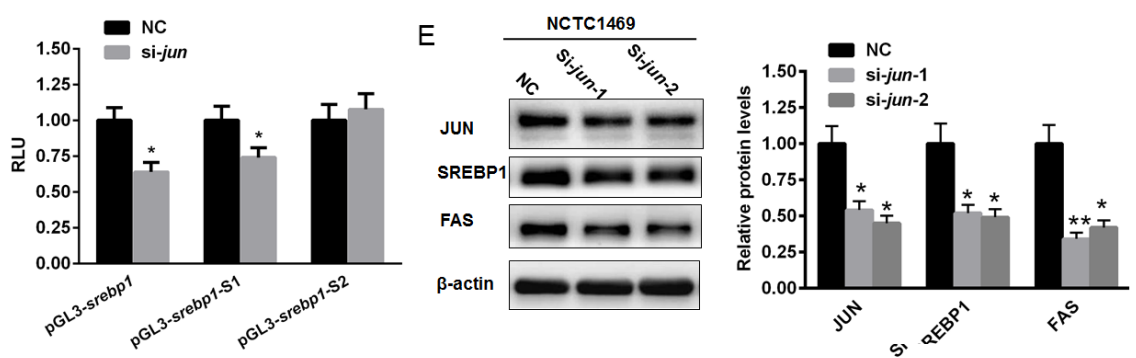

C
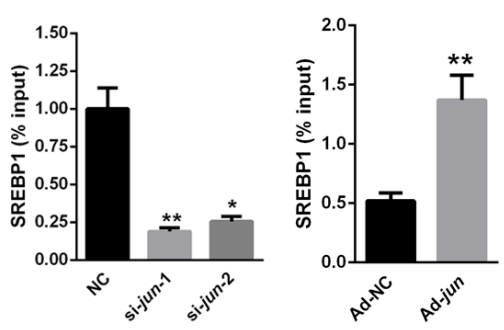

\section{D}
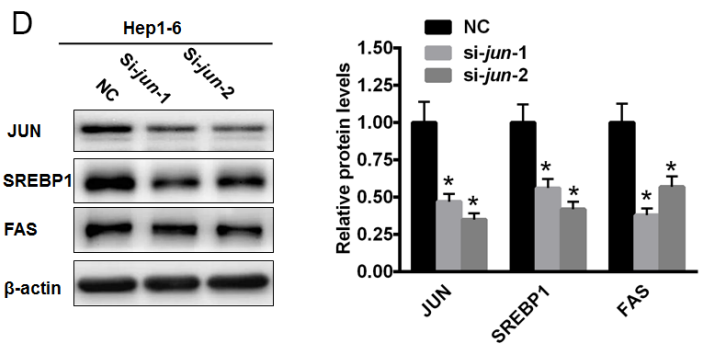

F NCTC1469

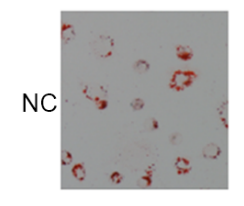

Hep1-6

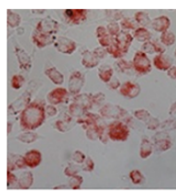

G

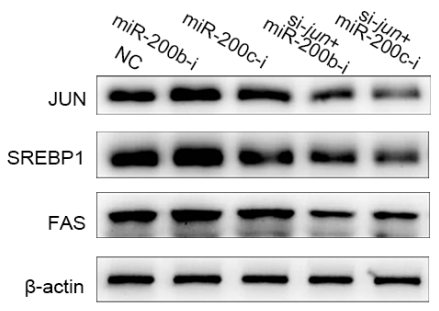

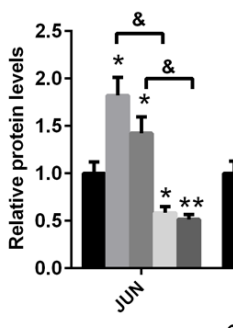

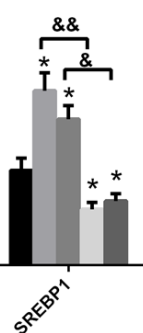

\section{\& 2 miR-200b-i}

miR-200b-i

si-jun+miR-200b-i

si-jun+miR-200c-i

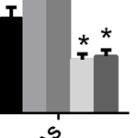

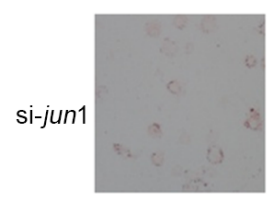
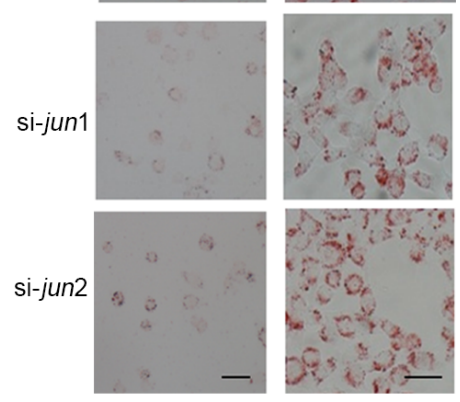

Figure 5: JUN activates lipid accumulation by activating the transcription of srebp1. (A) Schematic analysis of the potential JUN binding sites in the promoter region of murine srebpl gene. (B) Results of the luciferase reporter assay conducted in HEK293T cells co-transfected with siRNA specifically inhibiting jun (si-jun-1 or si-jun-2) and a luciferase reporter plasmid containing the promoters of either srebp1 (pGL3-srebp1), srebp1-S1 (pGL3-srebp1-S1) or srebp1-S2 (pGL3-srebp1-S2). (C) A srebp1 ChIP assay was performed in Hep1-6 cells transfected with si- jun/NC (upper panel) or ad- jun/N C (lower panel) for $48 \mathrm{~h}$. (D, E) Western blot analysis of SREBP1 and FAS expression in Hep1-6 and NCTC1469 cells transfected with si-jun-1 or si-jun-2. (F) Oil red O staining of Hep1-6 and NCTC1469 cells transfected with si-jun-1 or si-jun-2. (G) Western blot analysis of SREBP1 and FAS protein levels in Hep1-6 cells co-transfected with si-jun-1 and either the miR-200b inhibitor or the miR-200c inhibitor. The data represent the mean \pm SEM of three independent experiments. ${ }^{*} P<0.05$ and ${ }^{*} P P<0.01$ versus the control; ${ }^{\&} P<0.05$ and ${ }^{\&} \& P<0.01$ versus the miR-200b inhibitor or the miR-200c inhibitor. The bar represents $10 \mu \mathrm{m}$. 
A

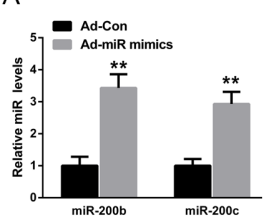

D

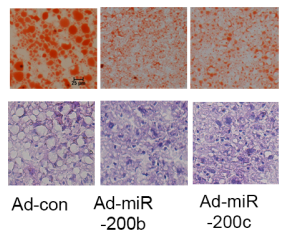

B

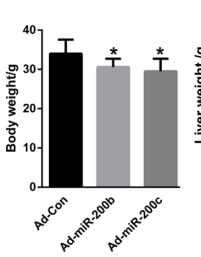

$E$

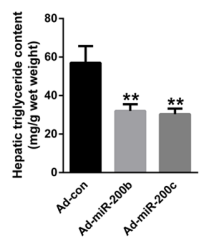

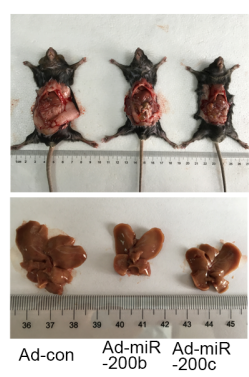

$\mathrm{F}$
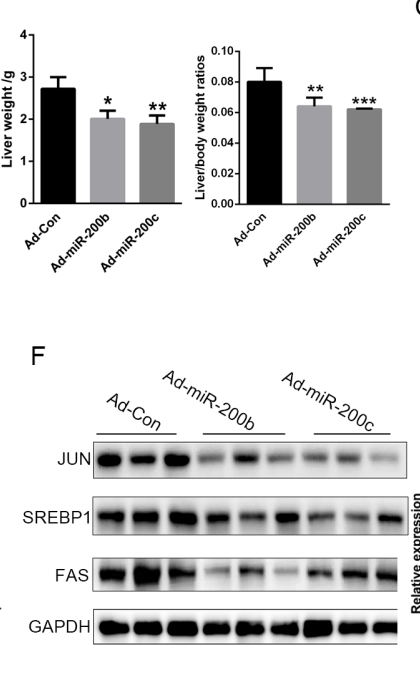

Ad-con $\quad$ Ad-miR Ad-miR

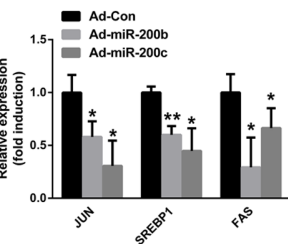

Figure 6: The up-regulation of miR-200b and miR-200c expression in the liver prevents hepatic lipid accumulation in mice fed a HFD. Six-to-eight-week-old male C57BL/6J mice fed a HFD for 8 weeks were injected with Ad-200b, Ad-200c or Ad-con through the tail vein and sacrificed 7 days later. (A) Levels of hepatic miR-200b and miR-200c. (B, C) Body weight, liver weight and liver weight-to-body weight ratio. (D) H\&E and Oil Red O staining of frozen liver sections. (E) Hepatic triglyceride content. (F) Western blots showing the hepatic expression of JUN, SREBP1 and FAS. The data represent the mean \pm SEM, $n=6$ mice. ${ }^{* *} P<0.01$ and ${ }^{* * *} P<0.001$ versus the control.

A

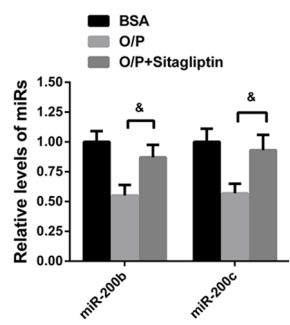

$E$
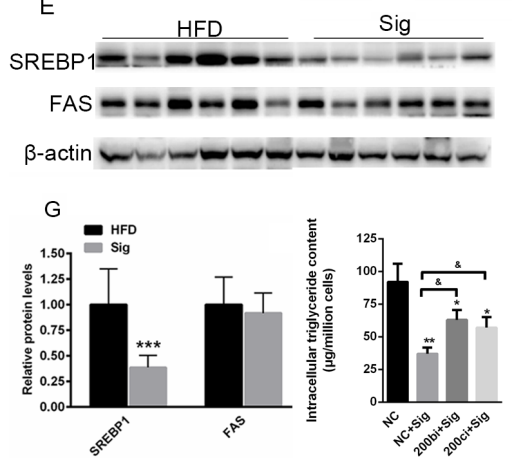

C
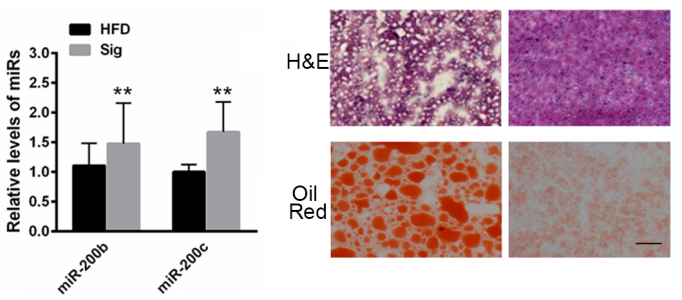

$\mathrm{F}$
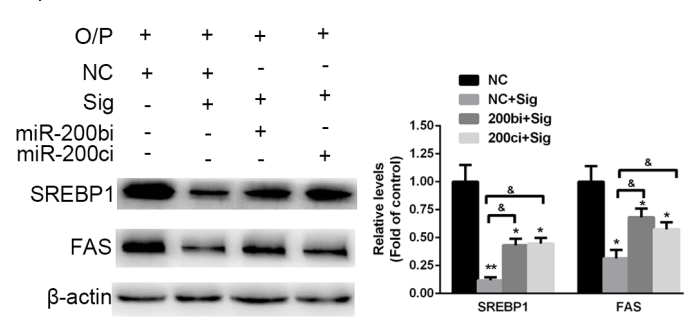

Figure 7: Elevated miR-200b and miR-200c expression is associated with sitagliptin-reduced hepatic lipid accumulation in mice fed a HFD. (A) Real-time reverse-transcription PCR analysis of miR-200b and miR-200c expression in Hep1-6 cells pre-treated with a mixture of oleic acid/palmitic acid (2:1, M/M) for $24 \mathrm{~h}$ and then treated with $1 \mu \mathrm{M}$ sitagliptin for $24 \mathrm{~h}$. (B) Quantification of miR-200b and miR-200c in the livers of HFD-fed mice treated with sitagliptin. (C) Oil red O staining and H\&E staining of the livers of HFD-fed mice treated with $3 \mathrm{mg} / \mathrm{kg} /$ day sitagliptin via i.g. for 8 weeks. (D) Measurement of hepatic triglyceride content in HFD-fed mice treated with sitagliptin. (E) Western blot analysis showing the expression of SREBP1 in the livers of sitagliptin-treated mice fed a HFD. (F, G) Western blot analysis showed that inhibition of miR-200b and miR-200c could partially rescue sitagliptin-induced reduced levels of SREBP1 and FAS, and decreased contents of intracellular triglyceride in Hep1-6 cells. The data represent the mean \pm SEM, $n=6$ mice or $n=3$ independent experiments. ${ }^{*} P<0.05$ and ${ }^{* *} P<0.01$ versus the control; $\& P<0.05$ versus $\mathrm{NC}+$ Sig. The bar represents $25 \mu \mathrm{m}$. 
miR-200c impaired lipid accumulation and the expression of lipogenic proteins such as SREBP1 and FAS. These results indicate that miR-200b and miR-200c suppressed hepatic lipogenesis.

In this manuscript, we identified jun as a target gene of miR-200b and miR-200c. Bioinformatics predictions revealed that there were conserved miR-200b and miR-200c binding sites in the 3'UTR of jun. The presence of these sites was validated using a luciferase reporter assay. JUN is correlated with a variety of biological processes including cell survival, cell proliferation, apoptosis and development [24, 33]. Through its transcriptional activity, JUN controls the expression of numerous genes involved in a variety of physiological processes. As a member of the basic leucine zipper motif (bZIP) family of proteins, JUN contains a basic DNAbinding domain and a leucine zipper region [34, 35]. JUN-protein interactions comprise a complex regulatory network that functions in a cell type-sepcific manner [36]. In the present study, we identified two putative JUN sites TGCGTCA and TGACTCA [37] in the promoter region of murine srebp1, raising the possibility that JUN may bind to the srebpl promoter. The results of a luciferase reporter assay showed that the knockdown of jun significantly reduced the relative luciferase activity of the srebpl promoter. Furthermore, the suppression of jun gene obviously reduced the expression of SREBP1, indicating that JUN activates SREBP1 expression.

Sitagliptin, a dipeptidyl peptidase (DPP)-4 inhibitor, was found to maintain glucose homeostasis by reducing the inactivation of incretin hormones such as glucagon-like peptide-1 (GLP-1) [40]. In vitro studies demonstrated that GLP-1 could enhance the activation of adenosine monophosphate kinase and peroxisome proliferator-activated receptor- $\alpha$, thereby inhibiting lipogenesis and increasing lipid oxidation, respectively $[41,42]$. In this study, we provide preliminary evidence that the elevated expression of miR-200b and miR-200c might contribute to the sitagliptin-induced reduction of hepatic lipid accumulation in HFD-fed mice. The treatment of HFD-fed mice with sitagliptin significantly impaired hepatic lipid accumulation and reduced the expression of SREBP1. Interestingly, the levels of miR-200b and miR-200c were increased in the livers of HFD-fed mice treated with sitagliptin. Moreover, knock down of miR-200b and miR-200c could partially rescue sitagliptin-induced reduced levels of SREBP1 and FAS, and decreased contents of intracellular triglyceride in Hep 1-6 cells

In conclusion, our findings may shed light on the specific molecular mechanism by which miR-200b and miR-200c are correlated with hepatic lipid metabolism. The reduced expression of miR-200b and miR-200c contributes to abnormal hepatic lipid accumulation by stimulating JUN expression and activating the transcription of srebp1.

\section{MATERIALS AND METHODS}

\section{Experimental animals}

Five-week-old male C57BL/6J mice were purchased from the Peking University Health Science Center. The C57BL/6J mice were fed a standard chow diet or a high-fat diet (HFD, D12451, 45\% kcal from fat, Research Diet, USA, http://www.researchdiets.com/ opensource-diets/stock-diets) for 10 weeks starting at approximately 5 weeks of age. The mice were housed in a temperature- $\left(20-24^{\circ} \mathrm{C}\right)$ and humidity-controlled (45-55\%) environment with a $12 \mathrm{~h} / 12 \mathrm{~h}$ light/dark cycle. Ten weeks after the initiation of the HFD, the C57BL/6J mice were treated with $3 \mathrm{mg} / \mathrm{kg} /$ day sitagliptin via i.g. for 8 weeks. These animals were sacrificed, and liver tissues were collected for further analysis.

All of the mouse procedures were approved by the Animal Ethics Committee at the Beijing Hospital.

\section{Human liver specimens}

The human liver biopsies and clinical procedures were performed with patient consent within the diagnostic workup of NAFLD. A total of 11 patients with NAFLD and 11 healthy control subjects were included in the study. Physical examinations, biochemical measurements, and body mass index (BMI) calculations were performed on all of the study participants. The application for patientderived materials was approved by the Research Ethics Committee of Beijing You-An Hospital, and written consent was obtained from all of the patients.

\section{Cell culture and treatment}

The NCTC1469 murine liver cell line and the Hep1-6 hepatic cancer cell line were purchased from American Type Culture Collection. Both of the cell lines were cultured in low-glucose Dulbecco's modified Eagle medium (DMEM) (Invitrogen, Carlsbad, California, USA) supplemented with 10\% horse serum (Hyclone, Logan, Utah, USA) or 10\% fetal bovine serum (Gibico, Grand Island, New York, USA), 100 units/ml penicillin (Invitrogen, Carlsbad, California, USA) and $0.1 \mathrm{mg} / \mathrm{ml}$ streptomycin (Hyclone, Logan, Utah, USA) at $37^{\circ} \mathrm{C}$ with humidified air and 5\% $\mathrm{CO}_{2}$. Sitagliptin (Sigma-Aldrich, St. Louis, Missouri, USA) was used at a final concentration of $1 \mu \mathrm{M}$.

\section{Oleic acid/palmitic acid (O/P) treatment}

$0.25 \mathrm{M}$ oleic acid (Sigma-Aldrich, St. Louis, Missouri, USA) and palmitic acid (Sigma-Aldrich, St. Louis, Missouri, USA) were dissolved in 100\% ethyl alcohol. Before use, the $0.25 \mathrm{M}$ oleic acid, the palmitic acid stock and 5\% BSA were incubated in a $60^{\circ} \mathrm{C}$ water 
bath for $10 \mathrm{~min}$. Then, $640 \mu \mathrm{l}$ of the oleic acid or $320 \mu \mathrm{l}$ of the palmitic acid stock were added drop-wise to $20 \mathrm{ml}$ of $5 \%$ BSA to obtain $8 \mathrm{mM}$ oleic acid and $4 \mathrm{mM}$ palmitic acid, respectively. Before the experiments commenced, the $8 \mathrm{mM}$ oleic acid and the $4 \mathrm{mM}$ palmitic acid were incubated in a $60^{\circ} \mathrm{C}$ water bath for $10 \mathrm{~min}$. Equal volumes of oleic acid and palmitic acid were mixed together. $300 \mu \mathrm{M}$ oleic acid/palmitic acid mixture $(2: 1, \mathrm{M} / \mathrm{M})$ was used to treat Hep1-6 and NCTC1469 cells for $24 \mathrm{~h}$.

\section{RNA isolation and real-time reverse- transcription PCR}

The miRNAs were isolated using TRIzol reagent (Invitrogen, Carlsbad, California, USA) according to the manufacturer's instructions. To detect and quantify the miRNAs, stem-loop reverse-transcription polymerase chain reaction (RT-PCR) was performed as previously reported [44]. The specific primers used in this study were described previously [23].

\section{Western blot analysis}

The cells or liver tissues were lysed with RIPA buffer (Solarbio, Beijing, China). Fifteen $\mu \mathrm{g}$ of protein was separated by $10 \%$ SDS-PAGE and further transferred onto a PVDF membrane (Millipore, Boston, Massachusetts, USA). The membrane was blocked with $8 \%$ non-fat dry milk and incubated with primary antibodies at $4^{\circ} \mathrm{C}$ overnight. The specific primary antibodies used in this study were antiSREBP1 (sc-366) (Santa Cruz, Dallas, Texas, USA), antiFAS (\#3180) (Cell Signaling, Boston, Massachusetts, USA), anti- $\beta$-actin (\#3700) (Cell Signaling, Boston, Massachusetts, USA) and anti-JUN (\#9165) (Cell Signaling, Boston, Massachusetts, USA). After washing three times with PBST, the membrane was incubated with the HRP-conjugated antirabbit or anti-mouse secondary antibody (Zhongshanjinqiao, Beijing, China. 1:5000) for $2 \mathrm{~h}$ at room temperature. Immunodetection was conducted using the ECL Plus detection system (Millpore, Boston, Massachusetts, USA) according to the manufacturer's instructions.

\section{Construction of adenovirus vectors}

The adenovirus vector containing jun (Ad-jun, VH836318) and the control vector (Ad-NC, cp0001) were purchased from ViGene BioSciences (Rockville, Maryland, USA). And the adenovirus vector expressing mimics of miR-200b or miR-200c was constructed by Genechem (Shanghai, China).

\section{Chromatin immunoprecipitation (ChIP)}

The Chromatin Immunoprecipitation Assay Kit was purchased from Millipore (Boston, Massachusetts, USA). Briefly, the nuclei extracted from cells transfected with Ad-JUN/NC or si-JUN/NC sonicated into $200-1000$ bp. Precleared chromatin was immunoprecipitated with anti-JUN and normal IgG antibodies according to the manufacturer's instructions. Immunocomplexes were added into $50 \mu \mathrm{l}$ of protein A/G-Sepharose beads and purified with Qiaquick (QIAGEN, Duesseldorf, German) PCR purification columns. The precipitated DNA was amplified with srebpl-specific primers. The primers specific to the JUN binding sites on the srebp 1 promoter were 5'-GTACCCTCAGGTCATACTGC-3' and 5'-AAGCTCTCTGGATTGCCTT-3'.

\section{Oil Red O staining}

Cells were treated with a mixture of oleic acid/ palmitic acid $(\mathrm{O} / \mathrm{P}, 2: 1, \mathrm{M} / \mathrm{M})$ for $24 \mathrm{~h}$ and washed three times with PBS (5 min each). The cells were then fixed with $4 \%$ paraformaldehyde for $10 \mathrm{~min}$ at room temperature. After dehydration with 100\% 1,2-propanediol for $5 \mathrm{~s}$, the cells were stained with pre-warmed Oil Red O for $30 \mathrm{~min}$ at $37^{\circ} \mathrm{C}$ and washed with $85 \%$ 1,2-propanediol. The cells were washed with distilled water and observed under a microscope.

\section{Triglyceride measurement}

The triglycerides in liver tissues or cells were extracted according to a modified method from Folch et al. [45]. Briefly, snap-frozen liver tissues (approximately $20 \mathrm{mg}$ ) were homogenized in $5 \mathrm{ml}$ of $1 \mathrm{M} \mathrm{NaOH}$ and extracted with $5 \mathrm{ml}$ of a chloroform/methanol $(\mathrm{v} / \mathrm{v}=2: 1)$ solution. The organic layer was dried under nitrogen gas and resolubilized in $1 \mathrm{ml}$ of chloroform containing 1\% Triton X-100. The extract was dried again and resuspended in $1 \mathrm{ml}$ of water. The triglyceride concentration was measured using a triglyceride enzymatic assay kit (ShenSuoYouFu Medical Diagnostic Products Co., Ltd., Shanghai, China).

\section{Histological analysis of tissues}

The liver samples were fixed in O.C.T. compound (Tissue-Tek, Japan) and sectioned at a thickness of $8 \mu \mathrm{m}$. The slides were stained with hematoxylin \& eosin (H\&E) or with Oil Red O as previously described [46, 47].

\section{Luciferase reporter assay}

For the luciferase assay, the jun $3^{\prime}$ UTR sequence was cloned into the pmirGLO plasmid as previously described [44]. The PCR cycling conditions were as follows: (1) a hot start step at $95^{\circ} \mathrm{C}$ for $10 \mathrm{~min}$; (2) 40 cycles at $95^{\circ} \mathrm{C}$ for $15 \mathrm{~s}$ and $55^{\circ} \mathrm{C}$ for $45 \mathrm{~s}$ and $(3) 72^{\circ} \mathrm{C}$ for $30 \mathrm{~s}$. The mutant was cloned using the Fast Mutagenesis System (TransGen Biotech, Beijing, China). The primers used to detect jun were 5'-CGAGCTCGGCCAAGGGTACACAAGATGG-3' 
and 5'-CCGCTCGAGCGGGGTCCCTGCTTTGAGAA TCA-3. jun -Mut-Forward: 5'-TTCGATCTCATTCAGTC GGAAAGGGGGG-3'; jun-Mut-Reverse: 5'- CCGACTGA ATGAGATCGAATGTTAGGTCCA-3'.

Before the luciferase reporter assay was carried out, $5 \times 10^{4}$ cells in $500 \mu \mathrm{l}$ of L-DMEM were seeded in each well of a 24-well plate and cultured for $18 \mathrm{~h}$. The cells were transfected with the modified firefly luciferase vector $(500 \mathrm{ng} / \mu \mathrm{l})$ mixed with the Vigofect transfection reagent (Beijing, China) according to the manufacturer's instructions. After $48 \mathrm{~h}$, the luciferase activities were measured using the dual-luciferase reporter assay system (Promega, Madison, Wisconsin, USA). Renilla luciferase activity was used as a normalization control.

\section{Promoter reporter analysis}

The PGL3 promoter vector and the PRL-TK vector were purchased from Promega Corporation. The promoter region of srebpl was amplified from the genomic DNA of Hep1-6 cells. The PGL3 promoter vector and the amplified fragments were digested with $\mathrm{XhoI} / \mathrm{KpnI}$ and purified by gel electrophoresis. The digested fragment was then inserted into the PGL3 vector up-stream of the SV40 promoter. HEK293T cells were co-transfected with the PGL3 plasmids and the PRL-TK vector using the VigoFect Transfection Reagent (Beijing, China). The cells were harvested and lysed $48 \mathrm{~h}$ posttransfection. The relative light units (RLU) were determined using the Dual-luciferase reporter assay system (Promega, Madison, Wisconsin, USA) according to the standard protocols. Normalized luciferase data (firefly/renilla) was compared with the empty pGL3promoter vector.

The RLU were determined using the dualluciferase reporter assay system (Promega, Madison, Wisconsin, USA) according to the standard protocol. The primers for amplification were as follows: srebp 1-F: 5'-GGGTACCCTTCTCTGCTCAACGAGGT CA-3', srebp1-R: 5'-CCTCGAGGTCTTTACCCTGTGC GGAA-3, srebpl-S1-F: 5'-GGGTACCCCTCAACGAGG TCAAAGA-3', srebp 1-S1-R: 5'-CCTCGAGGTACTCTG GTTTTGGTCAC-3', srebp 1-S2-F: 5'-GGGTACCCTTC TCTGCTCAACGAGGTCA-3' and srebp 1-S1-R: 5'-CTC GAGGTCTCAGCCCGACCGACAG-3'. The restriction sites for $\mathrm{KpI}$ and XhoI are underlined.

\section{Cell transfections}

The miR mimics, inhibitors and negative control (NC) were purchased from Genepharma (Shanghai, China), as were the siRNA-JUN and the non-specific siRNA (NC). The miR and siRNA transfections were performed using the HiPerfect transfection reagent (QIAGEN) as previously described [44]. Briefly, $1 \times 10^{6}$ cells in $2 \mathrm{ml}$ of L-DMEM culture medium supplemented with serum and antibiotics were seeded in 6-well plates. At the same time, the miR mimics, inhibitors or NC were mixed with HiPerfect transfection reagent (QIAGEN, Duesseldorf, Germany) and incubated at room temperature for $10 \mathrm{~min}$. The NCTC1469 and Hep1-6 cells were incubated with the complex for $48 \mathrm{~h}$.

\section{Statistics}

The data represent the mean \pm standard error of the mean (SEM). Differences were analyzed by Student's $t$-test, and statistical significance was set at $P<0.05$.

\section{ACKNOWLEDGMENTS AND FUNDING}

This work was supported by grants from the National Basic Research Program (973 program) of China (J.L. 2012CB517502, J. Q. 2014CB910503 and G.L. 2015CB964800) and by grants (J.L. 81270887 and X.H. 81270495 ) from the National Natural Science Foundation of China.

\section{CONFLICTS OF INTEREST}

The authors declare no conflicts of interest.

\section{REFERENCES}

1. Lazo M, Clark JM. The epidemiology of nonalcoholic fatty liver disease: a global perspective. Semin Liver Dis. 2008; 28:339-350.

2. Daniel S, Ben-Menachem T, Vasudevan G, Ma CK, Blumenkehl M. Prospective evaluation of unexplained chronic liver transaminase abnormalities in asymptomatic and symptomatic patients. Am J Gastroenterol. 1999; 94:3010-3014.

3. Tiniakos DG, Vos MB, Brunt EM. Nonalcoholic fatty liver disease: pathology and pathogenesis. Annu Rev Pathol. 2010; 5:145-171.

4. Lewis JR, Mohanty SR. Nonalcoholic fatty liver disease: a review and update. Dig Dis Sci. 2010; 55:560-578.

5. Serfaty L, Lemoine M. Definition and natural history of metabolic steatosis: clinical aspects of NAFLD, NASH and cirrhosis. Diabetes Metab. 2008; 34:634-637.

6. Plantier L, Besnard V, Xu Y, Ikegami M, Wert SE, Hunt AN, Postle AD, Whitsett JA. Activation of sterol-response element-binding proteins (SREBP) in alveolar type II cells enhances lipogenesis causing pulmonary lipotoxicity. J Biol Chem. 2012; 287:10099-10114.

7. Horton JD, Goldstein JL, Brown MS. SREBPs: activators of the complete program of cholesterol and fatty acid synthesis in the liver. J Clin Invest. 2002; 109:1125-1131.

8. Brown MS, Goldstein JL. The SREBP pathway: regulation of cholesterol metabolism by proteolysis of a membranebound transcription factor. Cell. 1997; 89:331-340. 
9. Bartel DP. MicroRNAs: target recognition and regulatory functions. Cell. 2009; 136:215-233.

10. Berezikov E, Cuppen E, Plasterk RH. Approaches to microRNA discovery. Nat Genet. 2006; 38:S2-7.

11. Osborne TF. Sterol regulatory element-binding proteins (SREBPs): key regulators of nutritional homeostasis and insulin action. J Biol Chem. 2000; 275:32379-32382.

12. Amemiya-Kudo M, Shimano H, Yoshikawa T, Yahagi N, Hasty AH, Okazaki H, Tamura Y, Shionoiri F, Iizuka Y, Ohashi K, Osuga J, Harada K, Gotoda T, et al. Promoter analysis of the mouse sterol regulatory element-binding protein-1c gene. J Biol Chem. 2000; 275:31078-31085.

13. Deng X, Zhang W, I OS, Williams JB, Dong Q, Park EA, Raghow R, Unterman TG, Elam MB. FoxO1 inhibits sterol regulatory element-binding protein-1c (SREBP-1c) gene expression via transcription factors Sp1 and SREBP-1c. J Biol Chem. 2012; 287:20132-20143.

14. Kornfeld JW, Baitzel C, Konner AC, Nicholls HT, Vogt MC, Herrmanns K, Scheja L, Haumaitre C, Wolf AM, Knippschild U, Seibler J, Cereghini S, Heeren J, et al. Obesity-induced overexpression of miR-802 impairs glucose metabolism through silencing of Hnflb. Nature. 2013; 494:111-115.

15. Krutzfeldt J, Stoffel M. MicroRNAs: a new class of regulatory genes affecting metabolism. Cell Metab. 2006; 4:9-12.

16. Lynn FC. Meta-regulation: microRNA regulation of glucose and lipid metabolism. Trends Endocrinol Metab. 2009; 20:452-459.

17. Xu Y, Zalzala M, Xu J, Li Y, Yin L, Zhang Y. A metabolic stress-inducible miR-34a-HNF4alpha pathway regulates lipid and lipoprotein metabolism. Nat Commun. 2015; 6:7466.

18. Gerin I, Bommer GT, McCoin CS, Sousa KM, Krishnan V, MacDougald OA. Roles for miRNA-378/378* in adipocyte gene expression and lipogenesis. Am J Physiol Endocrinol Metab. 2010; 299: E198-206.

19. Moore KJ, Rayner KJ, Suarez Y, Fernandez-Hernando C. The role of microRNAs in cholesterol efflux and hepatic lipid metabolism. Annu Rev Nutr. 2011; 31:49-63.

20. Chang L, Guo F, Huo B, Lv Y, Wang Y, Liu W. Expression and clinical significance of the microRNA-200 family in gastric cancer. Oncol Lett. 2015; 9:2317-2324.

21. Duhachek-Muggy S, Zolkiewska A. ADAM12-L is a direct target of the miR-29 and miR-200 families in breast cancer. BMC Cancer. 2015; 15:93.

22. Mahdavinezhad A, Mousavi-Bahar SH, Poorolajal J, Yadegarazari R, Jafari M, Shabab N, Saidijam M. Evaluation of miR-141, miR-200c, miR-30b Expression and Clinicopathological Features of Bladder Cancer. Int $\mathbf{J}$ Mol Cell Med. 2015; 4:32-39.

23. Dou L, Zhao T, Wang L, Huang X, Jiao J, Gao D, Zhang H, Shen T, Man Y, Wang S, Li J. miR-200s Contribute to Interleukin-6 (IL-6)-induced Insulin Resistance in Hepatocytes. J Biol Chem. 2013; 288:22596-22606.

24. Eferl R, Ricci R, Kenner L, Zenz R, David JP, Rath M, Wagner EF. Liver tumor development. c-Jun antagonizes the proapoptotic activity of p53. Cell. 2003; 112:181-192.

25. Hilberg F, Aguzzi A, Howells N, Wagner EF. c-jun is essential for normal mouse development and hepatogenesis. Nature. 1993; 365:179-181.

26. Akaslan SB, Degertekin CK, Yilmaz G, Cakir N, Arslan M, Toruner FB. Effects of sitagliptin on nonalcoholic fatty liver disease in diet-induced obese rats. Metab Syndr Relat Disord. 2013; 11:243-250.

27. Kato H, Nagai Y, Ohta A, Tenjin A, Nakamura Y, Tsukiyama H, Sasaki Y, Fukuda H, Ohshige T, Terashima Y, Sada Y, Kondo A, Sasaoka T, et al. Effect of sitagliptin on intrahepatic lipid content and body fat in patients with type 2 diabetes. Diabetes Res Clin Pract. 2015; 109:199-205.

28. Brunt EM. Pathology of nonalcoholic fatty liver disease. Nat Rev Gastroenterol Hepatol. 2010; 7:195-203.

29. Rhodes LV, Martin EC, Segar HC, Miller DF, Buechlein A, Rusch DB, Nephew KP, Burow ME, Collins-Burow BM. Dual regulation by microRNA-200b-3p and microRNA200b-5p in the inhibition of epithelial-to-mesenchymal transition in triple-negative breast cancer. Oncotarget. 2015; 6:16638-16652. doi: 10.18632/oncotarget.3184.

30. Senol O, Schaaij-Visser TB, Erkan EP, Dorfer C, Lewandrowski G, Pham TV, Piersma SR, Peerdeman SM, Strobel T, Tannous B, Saydam N, Slave I, Knosp E, et al. miR-200a-mediated suppression of non-muscle heavy chain IIb inhibits meningioma cell migration and tumor growth in vivo. Oncogene. 2015; 34:1790-1798.

31. Sun Y, Shen S, Liu X, Tang H, Wang Z, Yu Z, Li X, Wu M. MiR-429 inhibits cells growth and invasion and regulates EMT-related marker genes by targeting Onecut 2 in colorectal carcinoma. Mol Cell Biochem. 2014; 390:19-30.

32. Humphries B, Yang C. The microRNA-200 family: small molecules with novel roles in cancer development, progression and therapy. Oncotarget. 2015; 6:6472-6498. doi: 10.18632/oncotarget.3052.

33. Delmas A, Cherier J, Pohorecka M, Medale-Giamarchi C, Meyer N, Casanova A, Sordet O, Lamant L, Savina A, Pradines A, Favre G. The c-Jun/RHOB/AKT pathway confers resistance of BRAF-mutant melanoma cells to MAPK inhibitors. Oncotarget. 2015; 6:15250-15264. doi: 10.18632/oncotarget.3888.

34. Angel P, Allegretto EA, Okino ST, Hattori K, Boyle WJ, Hunter T, Karin M. Oncogene jun encodes a sequencespecific trans-activator similar to AP-1. Nature. 1988; 332:166-171.

35. Rauscher FJ 3rd, Voulalas PJ, Franza BR, Jr., Curran T. Fos and Jun bind cooperatively to the AP-1 site: reconstitution in vitro. Genes Dev. 1988; 2:1687-1699. 
36. Newman JR, Keating AE. Comprehensive identification of human bZIP interactions with coiled-coil arrays. Science. 2003; 300:2097-2101.

37. Bein K, Leight H, Leikauf GD. JUN-CCAAT/enhancerbinding protein complexes inhibit surfactant-associated protein B promoter activity. Am J Respir Cell Mol Biol. 2011; 45:436-444.

38. Ferreira DM, Afonso MB, Rodrigues PM, Simao AL, Pereira DM, Borralho PM, Rodrigues CM, Castro RE. c-Jun N-terminal kinase 1/c-Jun activation of the p53/ microRNA 34a/sirtuin 1 pathway contributes to apoptosis induced by deoxycholic acid in rat liver. Mol Cell Biol. 2014; 34:1100-1120.

39. Duarte N, Coelho IC, Patarrao RS, Almeida JI, PenhaGoncalves C, Macedo MP. How Inflammation Impinges on NAFLD: A Role for Kupffer Cells. Biomed Res Int. 2015; 2015:984578.

40. Ramadan WH, Kabbara WK. Sitagliptin/Simvastatin: a first combination tablet to treat type 2 diabetes and hypercholesterolemia - a review of its characteristics. Vasc Health Risk Manag. 2015; 11:125-132.

41. Ben-Shlomo S, Zvibel I, Shnell M, Shlomai A, Chepurko E, Halpern Z, Barzilai N, Oren R, Fishman S. Glucagon-like peptide-1 reduces hepatic lipogenesis via activation of AMP-activated protein kinase. J Hepatol. 2011; 54:1214-1223.

42. Svegliati-Baroni G, Saccomanno S, Rychlicki C, Agostinelli L, De Minicis S, Candelaresi C, Faraci G, Pacetti D, Vivarelli M, Nicolini D, Garelli P, Casini A,
Manco M, et al. Glucagon-like peptide-1 receptor activation stimulates hepatic lipid oxidation and restores hepatic signalling alteration induced by a high-fat diet in nonalcoholic steatohepatitis. Liver Int. 2011; 31:1285-1297.

43. Ferreira L, Teixeira-de-Lemos E, Pinto F, Parada B, Mega C, Vala H, Pinto R, Garrido P, Sereno J, Fernandes R, Santos P, Velada I, Melo A, et al. Effects of sitagliptin treatment on dysmetabolism, inflammation, and oxidative stress in an animal model of type 2 diabetes (ZDF rat). Mediators Inflamm. 2010; 2010:592760.

44. Guo J, Li M, Meng X, Sui J, Dou L, Tang W, Huang X, Man Y, Wang S, Li J. MiR-291b-3p induces apoptosis in liver cell line NCTC1469 by reducing the level of RNA-binding protein HuR. Cell Physiol Biochem. 2014; 33:810-822.

45. Folch J, Lees M, Sloane Stanley GH. A simple method for the isolation and purification of total lipides from animal tissues. J Biol Chem. 1957; 226:497-509.

46. Lee KH, Song JL, Park ES, Ju J, Kim HY, Park KY. AntiObesity Effects of Starter Fermented Kimchi on 3T3-L1 Adipocytes. Prev Nutr Food Sci. 2015; 20:298-302.

47. Kleiner DE, Brunt EM, Van Natta M, Behling C, Contos MJ, Cummings OW, Ferrell LD, Liu YC, Torbenson MS, Unalp-Arida A, Yeh M, McCullough AJ, Sanyal AJ, et al. Design and validation of a histological scoring system for nonalcoholic fatty liver disease. Hepatology. 2005; 41:1313-1321. 